\title{
Process analytical technology tools for process control of roller compaction in solid pharmaceuticals manufacturing
}

\author{
ALEŠA DULAR VOVKO ${ }^{1,2}$ \\ FRANC VREČER ${ }^{1,2, *}$ \\ ${ }^{1}$ Krka, d.d. \\ Novo mesto, 8501 Novo mesto, Slovenia \\ ${ }^{2}$ University of Ljubljana \\ Faculty of Pharmacy, 1000 Ljubljana \\ Slovenia
}

\begin{abstract}
This article presents an overview of using process analytical technology in monitoring the roller compaction process. In the past two decades, near-infrared spectroscopy, near-infrared spectroscopy coupled with chemical imaging, microwave resonance technology, thermal effusivity and various particle imaging techniques have been used for developing at-, off-, on- and in-line models for predicting critical quality attributes of ribbons and subsequent granules and tablets. The common goal of all these methods is improved process understanding and process control, and thus improved production of high-quality products. This article reviews the work of several researchers in this field, comparing and critically evaluating their achievements.
\end{abstract}

Keywords: process analytical technology (PAT), roller compaction, near-infrared (NIR) spectroscopy, near-infrared chemical imaging (NIR-CI), microwave resonance techno$\log y$, thermal effusivity

\section{INTRODUCTION}

Powders are often granulated to improve their flow properties, content uniformity and processability. Dry granulation is increasingly becoming a preferred granulation technique in the pharmaceutical industry $(1,2)$ because it makes manufacturing and processing of moisture and heat sensitive active pharmaceutical ingredients (APIs) possible (3-5). This process has also grown in popularity due to its cost and energy efficiency which results from fewer unit operations, shorter manufacturing times and the absence of the drying step (evaporation of solvents) $(1,6)$.

Roller compaction is a dry granulation process in which powder blends are compressed between two counter-rotating rollers to form dense ribbon compacts. Subsequently, ribbons are milled through screens to obtain granules, which are further mixed with excipients to form a compression or encapsulating mixture $(2,5,7,8)$.

Several factors can influence the compaction process. Physical and mechanical properties of raw materials, such as particle size and morphology, as well as process variables

\footnotetext{
*Correspondence, e-mail: franc.vrecer@krka.biz
} 


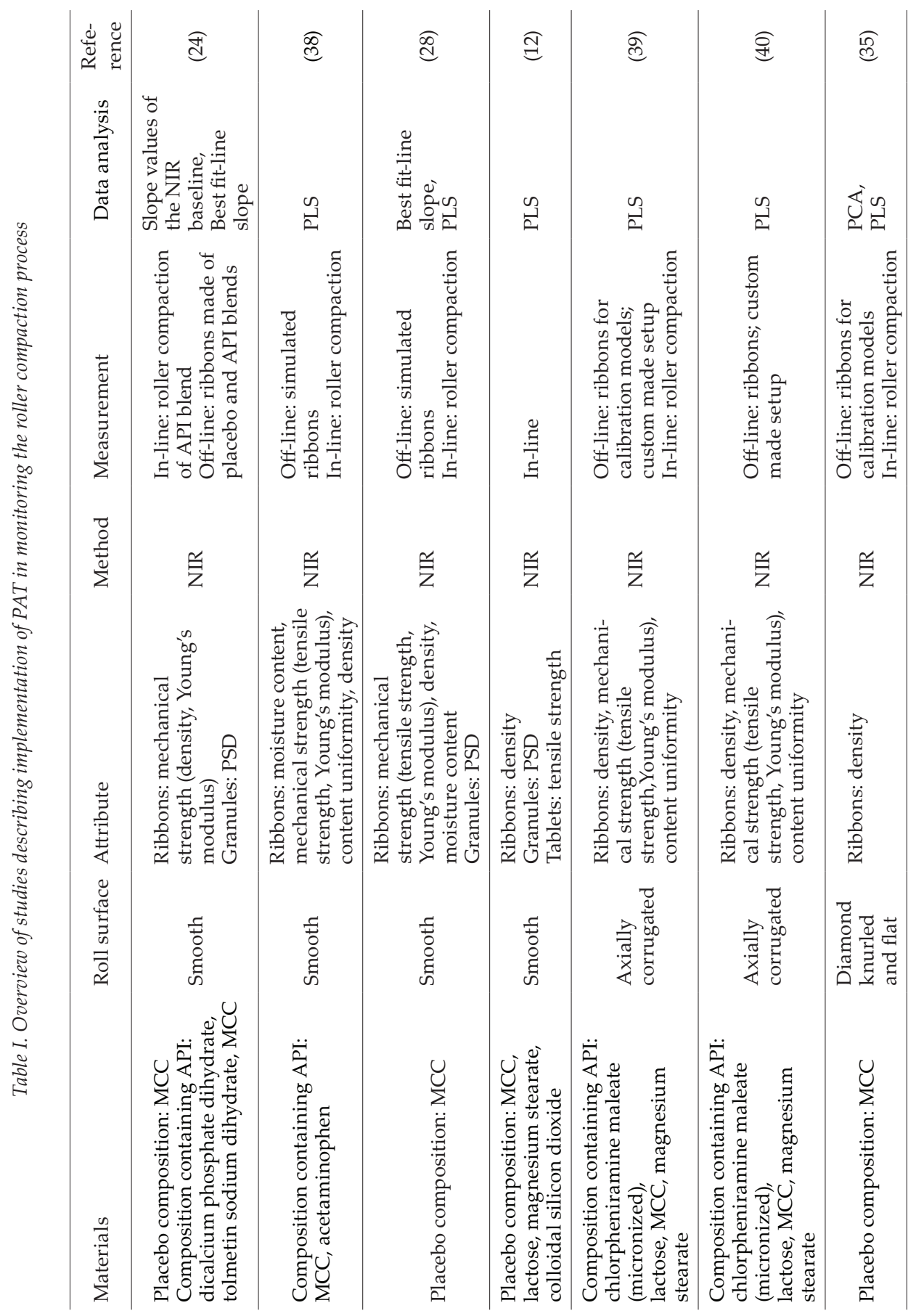




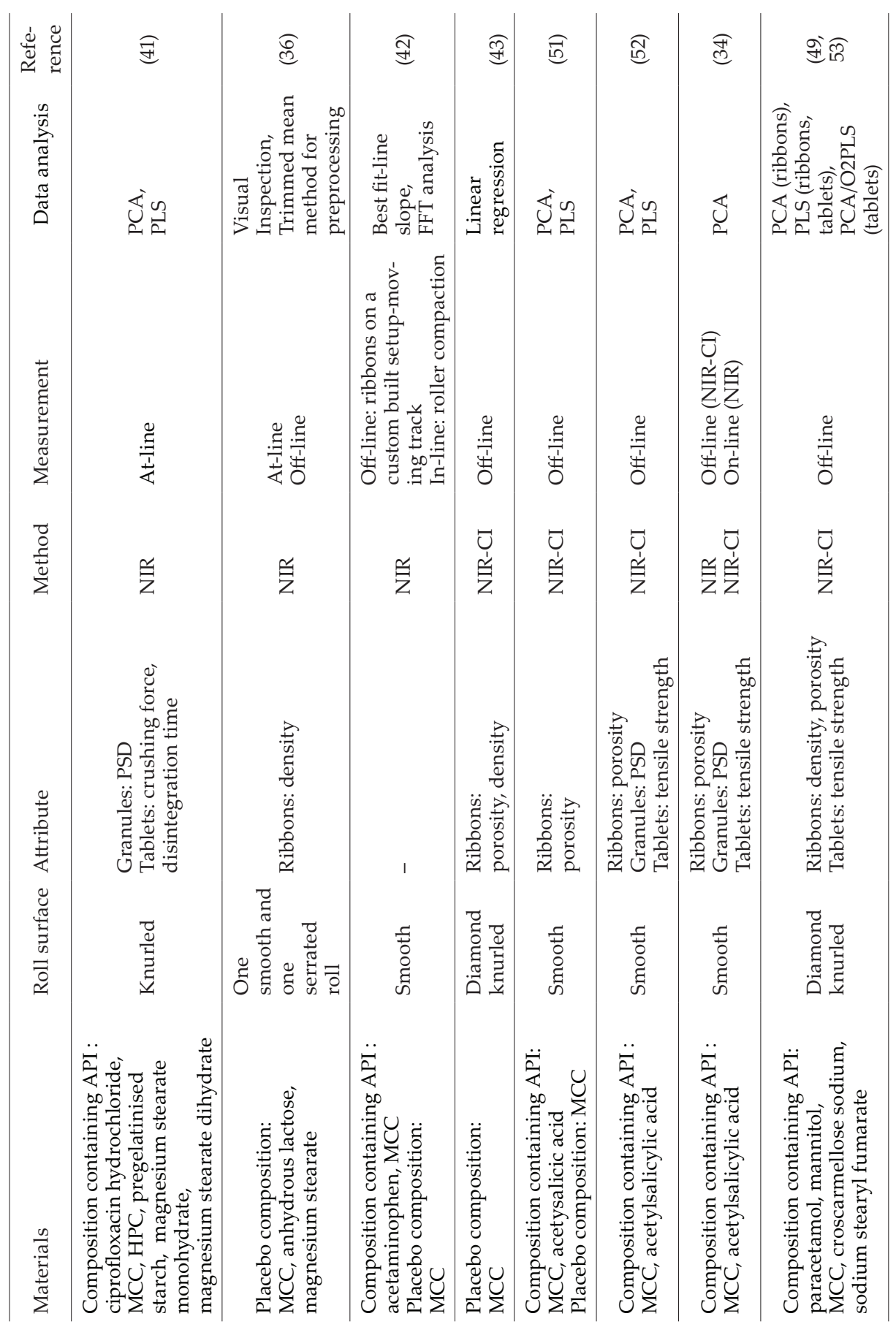




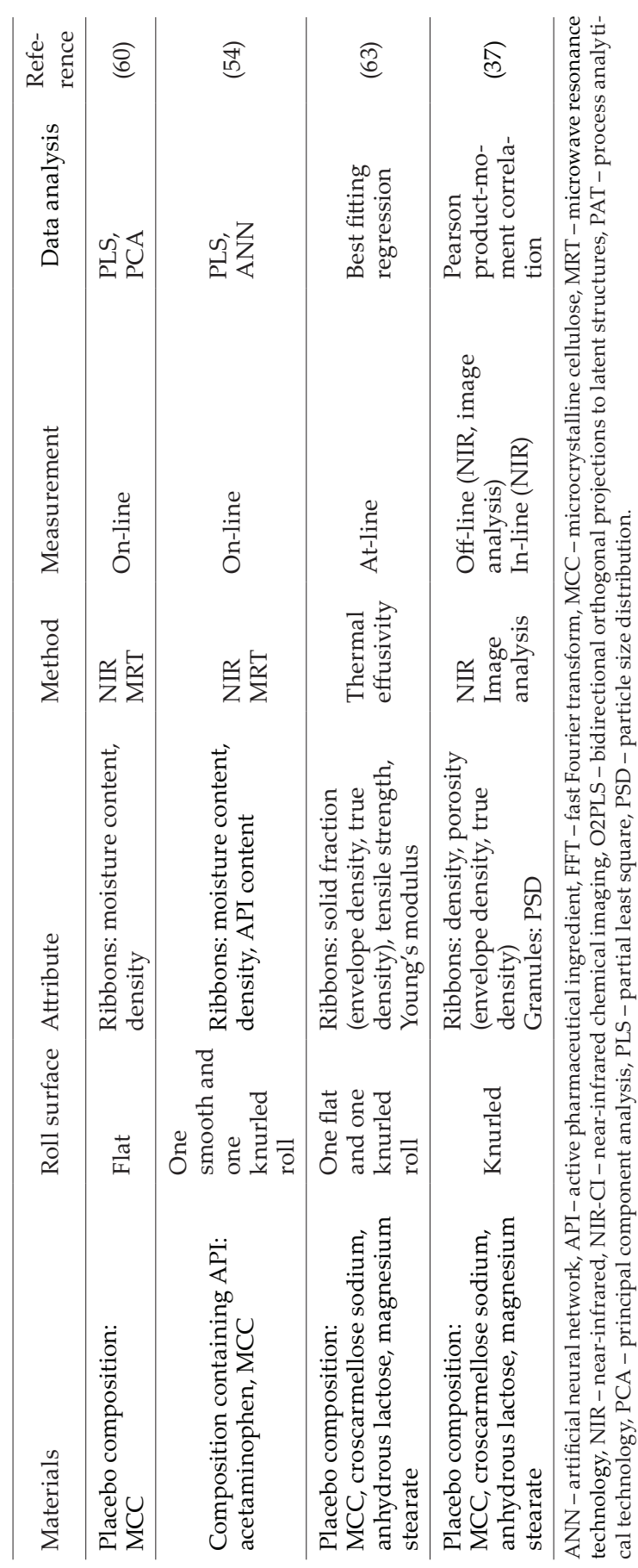

(feed screw speed, roll speed, roll force, roll gap and roll surface) and variations in the ambient moisture, may affect critical quality attributes (CQAs) of ribbons (such as density, porosity, strength and drug content) and consequently particle size distribution, flow characteristics, content uniformity, and compressibility of granules, compression mixture, and tablets. Therefore a better understanding of the roller compaction process is required to consistently ensure the desired and specified product quality (9-13).

The process analytical technology (PAT) initiative of the Food and Drug Administration (FDA) encourages the application of analytical techniques to reduce production cycle times and ensure final product quality by designing, analyzing, and controlling manufacturing through measurement of critical quality and performance attributes of raw and in-process materials and processes. Four types of process analytical measurements can be implemented: inline, with no removal of the sample; on-line, in which the sample is diverted from the main process, analyzed and may be returned; at-line, in which the sample is removed and analyzed close to the process and off-line, where sample is removed and analyzed away from the process (14).

This article reviews PAT tools implemented for monitoring the roller compaction process in the last 2 decades. This review article provides a system- 
A. Dular Vovko and F. Vrečer: Process analytical technology tools for process control of roller compaction in solid pharmaceuticals manufacturing, Acta Pharm. 70 (2020) 443-463.

atic insight into the PAT process analyzers, which have been (or have the potential to be) utilized for real-time or near real-time monitoring of CQAs. The working principle of analyzers is explained, and a critical overview of the applicability of individual techniques is discussed. In addition, an overview of the published studies is provided in Table I.

\section{APPLICATION OF NEAR-INFRARED SPECTROSCOPY IN MONITORING THE ROLLER COMPACTION PROCESS}

Near-infrared (NIR) spectroscopy investigates the absorption of electromagnetic radiation in the wavelength range from 700 to $2500 \mathrm{~nm}$ of the electromagnetic spectrum. In NIR spectroscopy, samples are irradiated with NIR light which leads to absorption due to molecular vibrations such as overtones and combinations of fundamental vibrations of polar bonds (15-19).

NIR spectroscopy is a rapid and nondestructive method, providing chemical, physiochemical and physical information whereby no or only little sample preparation is needed, and it is therefore widely implemented in the pharmaceutical industry as the PAT method (20-22).

Nevertheless, NIR spectroscopy also has its drawbacks. Calibration of spectral data with the reference method must be carried out prior to monitoring the process and models have to be constructed in order to extract the desired command variable (18). Upon collecting the NIR spectrum a characteristic wavelength range has to be selected and in most cases, the spectral data are pretreated. The objective of data pretreatment is to reduce variability unrelated to the attribute of interest so that the relevant changes can be modeled more effectively. Typical spectral preprocessing methods are standard normal variate (SNV), multiplicative scatter correction (MSC) and first or second derivatives. A univariate data analysis is possible, but due to the broad and highly overlapped absorption bands multivariate data analysis techniques, such as principal component analysis (PCA) or partial least squares (PLS), are preferably applied because they are able to use the entire spectral profile to extract useful information from the entire data set $(17,21)$.

PCA is an algorithm that transforms a set of correlated variables into a set of linearly uncorrelated ones. This type of analysis is a qualitative means for better understanding patterns in a complex data set. PLS regression is similar to PCA, and it is commonly used for resolving data sets into latent variables (LVs), which are principal components (PCs), whose linear combination approximates the original data to any desired degree of accuracy $(21,23)$.

A number of reports have investigated the use of NIR spectroscopy. These studies have taken advantage of the fact that the NIR absorption of a material is affected by the density of the material and therefore offers the potential to monitor roller-compacted ribbon density during production $(24,25)$. As ribbon density increases, there is less diffuse scattering of the NIR beam due to the reduction in air particle boundaries in the ribbon sample; less light reaches the detector, thus creating an apparently higher absorbance (see Fig. 1) $(26,27)$.

Moisture also influences the NIR spectrum, showing strong absorbance peaks at the 1,450 and 1,930 $\mathrm{nm}$ regions due to the specific bond stretching and vibrations (28). This characteristic allows accurate and precise water quantity evaluation, but at the same time, 
it can cause trouble when measuring other CQAs $(22,29)$. Pharmaceutical applications of NIR spectroscopy also include quantification of active ingredients and particle size analysis (30-33).

Gupta et al. (24) were the first to report the use of NIR spectroscopy for monitoring CQAs of the roller compaction process. They demonstrated the use of NIR spectroscopy to determine the strength (Young's modulus) of the ribbon and its ability to predict particle size distribution (PSD) obtained after milling the ribbons, which has been further investigated by Khorasani (34). NIR spectroscopy has also been utilized to detect changes in compaction behavior and mechanical properties due to the variability in the ambient moisture content (28). Later, Soh et al. (13) demonstrated that, in addition to NIR spectral slope, raw material properties, operating parameters and roll gap can also be utilized to construct robust models for granule and ribbon properties with good predictability. Their results have demonstrated the utility of roll gap and NIR spectral slope as process critical control parameters to better control the roller compaction process, specifically through ribbon density monitoring. Most of the recent studies performed by Acevedo et al. (35), Crowley et al. (36), and McAuliffe et al. (37), focused only on monitoring of ribbon density, demonstrating the suitability of NIR spectroscopy for evaluating ribbons of variable quality. The broad applicability of NIR spectroscopy to simultaneously determine CQAs of ribbons, such as drug content, density, tensile strength and Young's modulus of roller-compacted ribbons has also been demonstrated by Gupta et al. (38), Samanta et al. (39) and Quyet et al. (40). Their results demonstrate the possibility of identifying overcompressed or under compressed ribbons or ribbons containing a higher or lower percentage of API in real-time. Kona et al. (41) made an additional step forward by building multivariate models that were able to successfully predict the CQAs for granules (granule size) and tablets (crushing force and disintegration time).

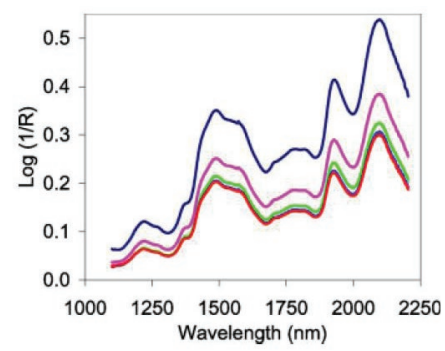

Fig. 1. NIR spectrum of $100 \%$ microcrystalline cellulose ribbons prepared at increasing roller compactor roll speeds (from top to bottom). The graph shows a decrease in NIR absorbance with increasing roll speed due to decreasing ribbon hardness as a consequence of decreasing mechanical strength and density of the ribbons $(24)^{1}$.

\footnotetext{
${ }^{1}$ Reprinted from Journal of Pharmaceutical Sciences, Vol. 93, A. Gupta, G. E. Peck, R. W. Miller and K. R. Morris, Nondestructive measurements of the compact strength and the particle-size distribution after milling of roller compacted powders by near-infrared spectroscopy, 1047-1053, 2004, with permission from Elsevier.
} 
A. Dular Vovko and F. Vrečer: Process analytical technology tools for process control of roller compaction in solid pharmaceuticals manufacturing, Acta Pharm. 70 (2020) 443-463.

The research articles listed above demonstrate that NIR can be successfully utilized for monitoring single- $(24,28,35)$ or multi-component $(12,36,37)$ placebo blends. Moreover, studies have also included mixtures containing API $(24,38-42)$, offering better insight into potential monitoring of the roller compaction process of real formulation.

Depending on the formulation properties, rolls with different surfaces - smooth (i.e. flat) or rough (i.e. knurled, axially corrugated, serrated, or fluted) may be used. Because the distance of the NIR probe and the ribbon surface is not constant for rough surface ribbons, process modeling and building the prediction models is believed to be more complex and has been shown to be inconsistent with the findings for flat surface ribbons. In the studies performed on flat-surface ribbons $(12,24,28,38)$, static spectral acquisition resulted in the best calibration models, whereas the dynamic sampling mode showed improved performance in a study performed on knurled rolls (39). The authors attributed this difference to the rough surface of the ribbon, on which the differences in the NIR light travel path during spectral acquisition were compensated for by the continuous movement of the ribbon surfaces, which simulated the movement of ribbons during real-time measurement. On the other hand, Acevedo et al. (35) compared the differences between models developed on different rolls within the same study. The results obtained by the flat roll validation experiment showed less accuracy when compared with the results obtained for the rough surface ribbons; however, the in-line results obtained for these models showed higher accuracy compared with the models developed with the spectra of rough surface ribbons. Nevertheless, offset between the predicted and reference values appeared for both models, which emphasizes the importance of matching the material analyzed by the reference method with the material analyzed in-line.

The authors of these articles used different experimental setup and data processing methods to obtain NIR spectra and achieve good predictive values of the NIR models for the selected CQAs. The first studies $(28,38)$ utilized simulated ribbons that were prepared under uniaxial compression. Attribute values predicted from the simulated ribbons showed large differences from the actual values measured during roller compaction (28). The authors did not take into account changes due to elastic recovery because they used samples for model creation which were stored for 24 hours before being analyzed. On the other hand, the real-time samples used for checking the model were fresh and were analyzed as they exited the rolls. In a study by the same authors (38), a Latin square experimental design was used to generate calibration samples, and it provided the best PLS model for predicting the selected ribbon attributes. However, large scatter was observed in the case of NIR-PLS predicted values for relative density, tensile strength, and Young's modulus, possibly due to the influence of non-compacted bypassing at the edges of the rolls during real-time monitoring, which was not present during data collection on the simulated ribbons. Obviously, the process influences ribbon properties, and therefore simulated ribbons do not perform as well as true samples. In order to improve the prediction ability of the method, the authors later used roller-compacted ribbons as calibration samples. NIR spectra were obtained by averaging spectra collected at selected ribbon intervals (37) by placing ribbons on a linear transverse setup (35), or by using a custom-built off-line setup (39, 40, 42). Samanta et al. (39) used a setup that consisted of a pair of parallel conveyor belts rotated by two rollers to capture off-line NIR spectra. Because the effect of the leakage of powder along the side seals was previously shown by Gupta et al. (38) to adversely affect the spectral acquisition, vacuuming of the dust particles was applied during the real-time monitored production. This was very effective and no marked effect 
in the NIR signal was observed. The agreement between the NIR predicted and reference values was good, which proves the good simulation of the real-time monitoring in the offline setup. Less variation was observed in the NIR-PLS predicted values for drug content compared to the values for tensile strength, envelope (bulk), and relative density. The authors attributed this to the time-dependent relaxation effect, which was significant for the off-line NIR signal collected spectra. Another possible explanation is the temperature effect because the real-time monitored compacts were warmed by frictional forces, whereas during off-line measurements the temperature of the compacts was already equilibrated with the room temperature, which was previously reported by Feng et al. (42).

In another study, Quyet et al. (40) proposed a simple performance evaluation using Equation (1) which was shown to estimate the relative performance for each calibration model in a more apparent manner than using the number of principal components (PCs), explained $X$ and $Y$ variances, root mean square error of calibration (RMSEC) or root mean square error of cross-validation (RMSECV) separately. The larger the performance index $\left(P_{\exp }\right)$ values the better the performance of the model.

$$
P_{\exp }=\frac{X+Y}{\sqrt{R M S E C}+\sqrt{R M S E C V}}
$$

Although the proposed theoretical model provided trends that did not match perfectly for all the NIR probes tested within the study, it can help distinguish between good and poor models and make possible recognition of the critical attributes that affect the collection of in-line spectral data.

Previous sections discuss the selection of the most representative calibration sample when assessing roller compacted ribbons. In the use of NIR spectroscopy in evaluating subsequent granules and tablets, the acquisition of spectra is less complex; on the other hand, on-line monitoring can be more technically challenging. Kona et al. (41) investigated tablets and granules by analyzing powder blends in glass scintillation vials, whereas the tablets were analyzed directly. The authors achieved good predictive values of the models for both granules and tablets. Khorasani et al. (34) proposed the use of an on-line custombuilt probe to record the spectra of the granules when the measuring window of the probe is covered with granules, whereby they could assess and monitor granule size after milling.

Apart from the selection of representative calibration samples and selecting optimal acquisition of spectra from the samples, spectral pre-processing, applying multivariate analysis to build an optimal calibration model, and correlating the spectral responses with reference values are also crucial in achieving good predictive values for new or unknown samples. In their first study, Gupta et al. (24) utilized the upward shift in the spectral baseline that occurs with increasing pressure, which has also been observed by other authors $(34,35,37,41,43)$. In order to quantify this upward shift, they selected the slope of the best fit line by applying regression analysis through the spectrum, which was first proposed by Kirsch and Drenner (44) as a mean to predict tablet hardness. Khorasani et al. (34) further observed that granule particle size also influences the NIR spectra, whereby larger granules have a higher slope and baseline shift compared to smaller granules. The authors proposed an algorithm based on a least-squares first-degree polynomial to calculate baseline slope values of the spectra for each granule batch. This method does not require pre-processing data and is a simple approach that has the potential to qualitatively - and with further development even quantitatively - assess and monitor granule size after milling. Pure 
spectrum analysis has also been utilized for monitoring ribbon density by Acevedo $e t$ al. (35), who compared this data analysis with PCA and PLS. All three methods showed a high potential for in-line monitoring of density distribution in roll compacts. However, pure spectrum analysis showed less accuracy at lower compaction force and for PLS models an offset between the prediction values was observed. The PCA model developed with off-line spectra correctly predicted the changes in a longer production run, therefore appearing as the most suitable method for real-time monitoring. However, other authors $(12,28$, $38,39,41)$ preferably proposed PLS modeling to predict the selected CQAs.

On the other hand, McAuliffe et al. (37) applied only standard normal variate (SNV) transformation as a preprocessing method in order to reduce the effect of the physical variation of the ribbon due to a knurled roller surface. Off-line NIR measurements were compared to off-line ribbon envelope density measurements of roller-compacted ribbons and in-line NIR measurements obtained using the multipoint NIR spectrophotometer Multieye $^{\mathrm{TM}}$ NIR probe. A good correlation with an acceptable Pearson correlation coefficient has been observed between in-line and off-line measurements. The results demonstrate thatMultiEye $\mathrm{T}^{\mathrm{TM}}$ could be used as a PAT tool in monitoring continuous unit operations at a commercial scale; however, validation is required to demonstrate accuracy after a long period of manufacturing on the production scale.

Multieye ${ }^{\mathrm{TM}}$ NIR has also been utilized in a recent study highlighting the challenge of interpreting a noisy spectral data set (36). The authors showed that a non-subjective pretreatment trimmed mean method is capable of screening for erroneous probe readings and is an effective method for discarding spectral results due to ribbons of variable quality, which may cause poor calibration of the model or incorrect analysis of the results.

However, prediction of new or unknown samples may also be a challenge due to variability of the environment of the process, NIR sensor sensitivity, and light stray variation (42), or due to site/instrument variability (41), which has been addressed by the studies presented below.

Feng et al. (42) proposed a method for reducing variability by utilizing a new in-line control kit using NIR spectroscopy together with a fast Fourier transform (FFT) analysis. FFT on NIR monitoring of the roller compaction process could determine the source of variability, materials or motions of the rolls. The in-line control kit minimized the variability due to the motion of the rolls. It included reduced powder floating around the ribbon and offered strict control of the movement of ribbon, which reduced the light stray effects of the NIR source and minimized the deformation and relaxation effects.

Kona et al. (41) were the first to investigate variability due to site/instrument variability. To test the model validity, calibration models were applied to external batches that were manufactured at different locations, using the same model roller compaction and process parameters. Batches with the same formulation, materials and processing parameters made on the second roller compactor had statistically significant different particle sizes from Site 1 particles, which led to quality changes of tablets (different crushing force and disintegration time). The difference between the sites was demonstrated by the PCA analysis of granules and tablets from both sites. In the authors' opinion, the differences could occur due to roller compactor calibration differences, resulting in different actual roller compaction process parameters; however, the reason was not examined and verified by the authors. 
The studies listed above show the scope of NIR spectroscopy applicability in monitoring and controlling the roller compaction process. Nevertheless, this PAT approach still has many aspects that must be investigated in greater detail. The studies demonstrate that it can be used for the monitoring of single- or multi-component mixtures; however, studies preferably used excipients for roller compaction, that were capable of producing uniform ribbon samples. The applicability of less-investigated excipients and high- or low-dose model active ingredients should be clarified in the future. Namely, different physical properties of ingredients could result in split, broken, or curved ribbons, which may cause noisy spectral data. Considering the complexity of the process, utilization of novel pretreatment methods and more complex multivariate approaches should also be investigated in greater detail. The fundamental mechanisms dominating variabilities - such as the sensitivity of the probe, ribbon sample presentation to the NIR probe, and control of the environmental conditions - have been addressed, however, this area demands further experimental work. Our review shows the applicability and validity of NIR for monitoring and predicting chemical and physical CQAs of the roller compaction process, such as ribbon density, moisture, content uniformity, and granulate particle size distribution. The applicability of NIR in predicting the CQAs of the granulate and subsequent tablets also deserves further attention. Most studies focused on the production of ribbons and ribbon evaluation. The possibility of realtime monitoring of the ribbon milling process and milling-related characteristics should also be investigated. Although the experiments in the currently available studies were performed on a smaller scale, the industriability may be achieved with this method. However, this field needs to be investigated in detail, and this is expected to be complex and difficult because Kona et al. (41) showed that even site variability may be an issue.

\section{Application of near-infrared chemical imaging in monitoring the roller compaction process}

Near-infrared chemical imaging (NIR-CI) is a combination of conventional NIR spectroscopy and digital imaging. NIR-CI is a rapid and nondestructive method that allows the collection of many thousands of NIR spectra (chemical information) as a function of the position in the sample. These spectra can be compiled into an image (spatial information) (32). The spectral and spatial information are simultaneously recorded and stored as a three-dimensional (3D) dataset or hyperspectral data cube, which adds spatial information to the spectral information. The $x$ and $y$ axes represent the spatial or pixel information and $z$ is the wavelength axis in the spectral measurement. Initially, data processing must be implemented in order to unfold the 3D hyperspectral data cube into a two-dimensional (2D) data matrix in which each row is a spectrum (sample) related to one of the pixels. The 2D data matrix is then processed by the selected chemometric model and refolded into the 3D hyperspectral data cube, where the chemical or physical image can be obtained. The images provide distribution information from the sample surface $(17,45)$. The information depth, which is the depth of the sample contributing to the measured reflected radiation, depends on the wavelength, sample presentation, instrument settings, and physical and chemical properties of the sample $(46,47)$. Clarke et al. (46) found an exponential relationship of NIR light penetration with wavenumber. In their study the information depth varied from 777 to $109 \mu \mathrm{m}$; however, half of the contribution to any spectrum came from the top surface depth in a range between 180 and $25 \mu \mathrm{m}$ (46). NIR-CI offers a larger effective sample size because it acquires chemical information across larger sample areas using 
A. Dular Vovko and F. Vrečer: Process analytical technology tools for process control of roller compaction in solid pharmaceuticals manufacturing, Acta Pharm. 70 (2020) 443-463.

larger sensor arrays in comparison to NIR spectroscopy probes. In addition, spatial and spectral information can enhance process understanding and improve process data interpretation (48).

Analysis of NIR-CI data offers qualitative and quantitative insight into the chemical and physical characteristics of heterogeneous samples (32) and provides a process monitoring solution as demonstrated in the following studies.

Lim et al. (43) were the first to report the use of NIR-CI to evaluate porosity variations of roller-compacted ribbons along a ribbon's width and length. They related the mean spectral absorbance at a single wavelength for different ribbons to their reference porosity values. Later Souihi et al. (49) have expanded the investigated field of CQAs to the chemical mapping of the API distribution in the subsequently produced tablets. Both studies $(43,49)$ have demonstrated the ability to characterize differences in porosity as a function of position on the ribbon, where the ribbon density is lower at the edges and higher at the center. When assessing axial porosity variation, the intensity map showed sinusoidal variation along the ribbon, which is a result of the periodicity of the powder movement by the feed screw, as was previously reported by Guigon and Simon (50). NIR-CI was also utilized in monitoring the uniformity, compaction pressure, and tensile strength of the tablets because the method can visualize the spatial distribution of the compaction pressures on the surface of the tablets (49).

Khorasani et al. $(34,51,52)$ have further demonstrated the feasibility of the NIR-CI use for visualization and prediction of porosity distribution. In addition, they have utilized NIR-CI to map the API distribution in the ribbons prepared at different process settings and in the corresponding tablets (52). Each pixel in the score image contains a score value, which can be related to a porosity value per the PCA model. The trend for $50 \%$ and $90 \%$ was consistent with the observed relationship at which lower ribbon porosity leads to larger granules. However, the trend with a $10 \%$ fraction was not clear, which indicates a limitation of the NIR-CI prediction.

The above-mentioned studies were performed on placebo $(43,51)$ and formulations containing APIs $(34,49,51,52)$ using flat $(34,51,52)$ and rough $(43,49)$ surface rolls. The diamond knurl surface of rolls was shown to cause porosity variation among the raised and depressed regions. In the ribbons compacted with the depressed area, more material per volume is deposited than on the parts of the ribbon compacted by the raised area of the rolls. As a result, the regions compressed by the raised area of the rolls showed lower porosity compared to the depressed area (see Fig. 2) (49). In addition to density variation due to the surface of the ribbon, the ribbons also exhibited axial variation of porosity, possibly due to the feeding mechanism as mentioned in the previous section.

On the other hand, Khorasani et al. $(34,51,52)$ have demonstrated that the porosity of flat ribbons is also not homogeneous. Less porous ribbons, manufactured at higher roll pressures, exhibited higher heterogeneity, which can also be connected to the feeding process.

The studies listed above proposed different modeling approaches for data analysis. Lim et al. (43) successfully utilized a simple linear regression model; however, a more complex composition demanded the use of multivariate methods. Souihi et al. (49) have utilized the PCA technique in assessing porosity variation across a roll-compacted ribbon and PLS models to predict the local compaction pressure and tensile strength information 


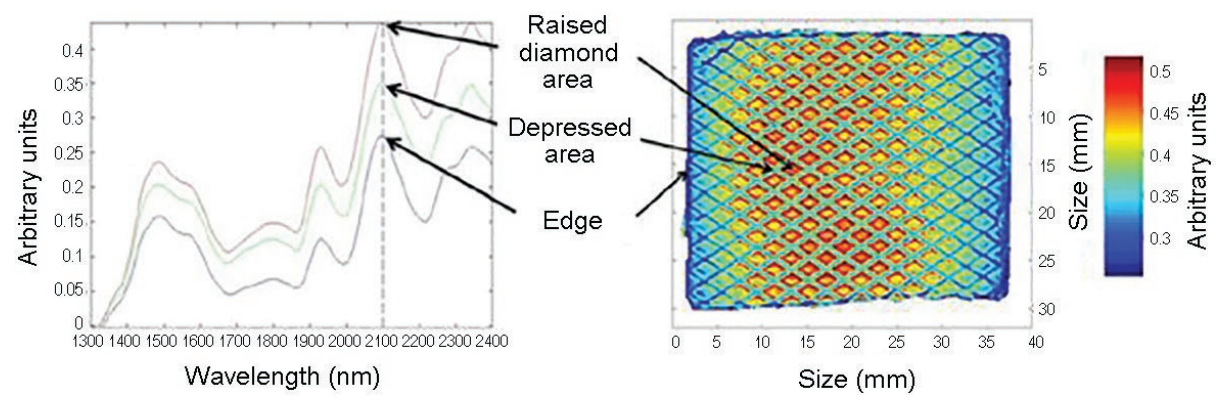

Fig. 2. Color mapping of the roller-compacted ribbon, showing variation in porosity. The red spectrum corresponds to regions within the raised area exhibiting the lowest porosity, the green spectrum to the regions within the depressed area with higher porosity, and the blue spectrum to regions around the edge, where porosity was the highest $(43)^{2}$.

from the surface of consecutively produced tablets. A PCA and O2PLS (bidirectional orthogonal projections to latent structures) model were further used in the analysis of the texture of chemical tablet images. The results of chemical imaging indicate that the homogeneity of the API can be correlated with the roll-compaction process parameters (53). Khorasani et al. (51) have confirmed the applicability of the porosity prediction by using NIR-CI in combination with PCA and PLS regression. In their further articles, Khorasani et al. $(34,52)$ demonstrated that the PCA model can be used as a surrogate method for estimating the ribbon's porosity. PLS regression was further used to visualize and predict the API content and distribution in ribbons and corresponding tablets.

The studies presented above confirm the potential of NIR-CI and NIR spectroscopy for process monitoring and control of roller compaction. NIR spectroscopy averages spectral information from the entire data collection area, producing a single result. Because the variation in chemical and physical properties over an area may also influence the quality of the resulting product, a bulk characterization method may not be able to adequately probe the sample, and therefore NIR-CI is considered a more advantageous tool.

NIR-CI provides qualitative and quantitative insight into the chemical and physical characteristics of the ribbons, such as ribbon density, spatial porosity distribution, API concentration and API distribution, as well as the CQAs of the subsequently manufactured granules and tablets. However, additional experimental input will be needed to confirm the applicability of real formulations because most of the studies were performed on abbreviated formulations such as individual fillers or filler/API blends. Because the process and data obtained are complex, the use of novel statistical approaches should also be investigated. Process analyzers will also have to be improved to be fast and precise enough to allow real-time analyses and in-line process monitoring on a pilot and industrial scale.

Reprinted from International Journal of Pharmaceutics, Vol. 410, H. Lim, V. S. Dave, L. Kidder, E. N. Lewis, R. Fahmy and S. W. Hoag, Assessment of the critical factors affecting the porosity of roller compacted ribbons and the feasibility of using NIR chemical imaging to evaluate the porosity distribution,1-8, 2011 with permission from Elsevier. 
A. Dular Vovko and F. Vrečer: Process analytical technology tools for process control of roller compaction in solid pharmaceuticals manufacturing, Acta Pharm. 70 (2020) 443-463.

\section{APPLICATION OF MICROWAVE RESONANCE TECHNOLOGY IN MONITORING THE ROLLER COMPACTION PROCESS}

Microwave resonance technology (MRT) is a novel approach for measuring material's physical and chemical (54) properties. It is based on the interaction between water molecules and the electromagnetic field. The measuring frequency of the MRT sensor is defined by the resonance wavelength of the microwave inducing resonator (18). When a material is passed over a microwave resonance sensor, its resonance frequency decreases, and the half-width of the resonance curve increases due to an increase in the signal's attenuation (55). The magnitude of these changes can be correlated with the water content and density of the samples, and therefore the method is appropriate for accurate measurements of these two parameters $(56,57)$.

The method may also be implemented as PAT because it does not require sample preparation and it provides real-time data due to their fast acquisition and processing times. The technique allows reflection and transmission measurements in the microwave frequency region without contact with the sample and it also makes possible work without sophisticated chemometric software $(58,59)$.

Austin et al. (60) compared the on-line determination of roller-compacted ribbons' envelope density and moisture content with microwave resonance and NIR spectroscopy. With MRT a root mean square error (RMSE) for determinating density was $0.044 \mathrm{~g} / \mathrm{cc}$. When using NIR spectroscopy, the RMSE of the calibration was similar to that found by other authors $(28,35)$ and was approximately twice as high as that achieved with MRT. This can be attributed to the fact that by using MRT the amount of mass present in the field can be directly related to the magnitudes of the microwave sensor, whereas physical properties such as density need to be filtered from chemical effects using a technique such as PCA when using NIR spectroscopy. When monitoring the moisture content, the RMSE obtained by microwave sensing was also approximately half of the error seen within NIR analysis. Microwave sensors are very sensitive to moisture due to a large contrast between the dielectric properties of water and most solid materials, and therefore they are capable of detecting water concentrations in the ppm range, whereas NIR detecting schemes must use complex chemometric software and spectral pretreatments to remove physical effects from spectra before they can accurately monitor chemical compositions.

Gupta et al. (54) further introduced a novel microwave sensor for the on-line measurement of roller-compacted ribbon's critical attributes with improved accuracy. The authors made a step forward in this study with the use of mixed composition blend by combining one smooth and one knurled roll and especially by introducing measurement of API concentration. The pretreatment of the MRT signal was also first presented in this study (54) and has proven to be important in microwave data analysis. Microwave and NIR data were analyzed using PLS regression and artificial neural networks (ANNs). The results from the PLS analysis show that microwave resonance sensing performs better than NIR sensing for ribbon density, moisture content and API concentration. From the results of the ANN modeling, it can also be seen that the ANN modeling of the MRT results performs better than the modeling of NIR results for each quality attribute. PLS and the ANN yield similar results with MRT and NIR data, except for moisture content, which is better predicted using the ANN, which possibly indicates the presence of non-linear trends in the data that have not been captured by the PLS algorithm. 
The results of both studies $(54,60)$ indicate that MRT is a promising tool for measuring CQAs of roller-compacted formulations such as density, moisture content and API concentration of the ribbons and for providing useful data during the roller compaction process in real-time. Although microwave resonance was shown to be more accurate than NIR spectroscopy for determining moisture content and density in roller-compacted ribbons, and it also does not require the use of sophisticated chemometric methods, and the sensors can be cheaply designed and fabricated, it does not provide as accurate information on chemical composition of the sample as NIR spectroscopy if it is correctly calibrated. However, it can provide precise insight into the sample composition for the selected attributes because the microwave sensor analyses the entire ribbon mass, whereas the NIR sensor only sees a fraction of the ribbon width and is limited to shallow penetration into the ribbon, whereas microwave resonance can penetrate several $\mathrm{cm}$ into the material.

The applicability of the method should also be confirmed with further studies on various formulations on different levels; that is, from the laboratory scale to the pilot and industrial scales.

\section{APPLICATION OF THERMAL EFFUSIVITY IN MONITORING THE ROLLER COMPACTION PROCESS}

Thermal effusivity is a material property that combines thermal conductivity, density, and heat capacity and can differentiate between solids, liquids, and powder components in a system based on heat-transfer properties (e.g., in a pharmaceutical formulation) (61). Powders have different effusivity readings depending on their ability to transfer heat through and between the particles. Effusivity is also a function of the particle size, shape, density and moisture content of material (62).

Thermal effusivity is a nondestructive technique and it does not require intense data pretreatment and chemometric data analysis for method development. It, therefore, offers the potential for implementation as a PAT tool $(61,62)$.

Ghorab et al. (63) have evaluated the feasibility of using thermal effusivity for inprocess monitoring of roller compaction by examining the relationship between physical characteristics of compacted ribbons and their thermal effusivity. Thermal effusivity, a solid fraction (defined as the ratio between ribbons' envelope density and the bulk density of the material), tensile strength, and Young's modulus of ribbons made of MCC, anhydrous lactose and placebo formulations containing various ratios of MCC to lactose were determined at different compaction pressures. The correlation between effusivity and selected physical properties was strong; however, it was formulation-dependent. The differences in the results obtained for mainly MCC and lactose formulations could be attributed to different deformation behavior of these two materials under compression. Lactose particles deform by brittle fracture, which results in a significantly increased number and size of interparticle contact points, causing a change in thermal conductivity in addition to a density change. Thus, the change in lactose effusivity with compaction pressure is dependent not only on the increase in solid fraction but also on the size of the fractured particles. On the other hand, MCC particles exhibit plastic deformation, in which the change in contact points between the particles is accompanied by consolidation and might not have a significant effect on thermal conductivity. Thus, the change in MCC effusivity with an increase in compaction pressure was mainly dependent on the density change. 
The results of the study demonstrate the possibility of using effusivity as a tool for monitoring roller compaction; nevertheless, there are some limitations regarding ribbon properties. Only the flat face of the compact can be analyzed because the knurled side gives erroneous readings due to the air trapped between the knurls and the sensor. Incorrect readings were also obtained for long ribbons because their curvature left part of the sensor uncovered, and therefore only short segments can be analyzed.

Moreover, further instrumental improvement is also required for on-line monitoring because effusivity readings also require a few seconds of static direct contact between the sensor and the ribbon, and an additional 30 seconds of sensor cooling time. Therefore, extensive research and development will be needed before thermal effusivity becomes a PAT tool for monitoring the roller compaction process.

\section{APPLICATION OF IMAGE ANALYSIS IN MONITORING THE ROLLER COMPACTION PROCESS}

An adequate PSD of the granulate in pharmaceutical formulations has an important impact on the product's safety, efficacy, and quality, and it represents a CQA in a number of pharmaceutical operations, including roller compaction. Therefore, monitoring and controlling PSD is essential to the pharmaceutical industry (64).

Although NIR spectroscopy and NIR-CI can be utilized for monitoring PSD (24, 34, 51), the most frequently applied PAT process sizing techniques include focused beam reflectance measurements (FBRM), spatial filtering velocimetry (SFV), photometric stereo imaging, dynamic image analysis and the flash imaging technique because they provide detailed insight into particle size and shape. The last two techniques have also been used in monitoring the roller compaction process.

Dynamic image analysis is a rapid non-invasive method that is recently becoming frequently used in the pharmaceutical industry for the evaluation of particle size and shape (65). The method provides images of fast-moving particles and is sensitive to differences in size and shape characteristics, which is especially suitable in sizing non-spherical particles $(66,67)$.

The flash imaging technique also allows the determination of the size and shape of moving particles. The apparatus creates a powerful short light pulse such that sharp images of particles are captured. Particles are illuminated by various light-emitting diodes (LEDs) from different angles and the color on the surface is captured in an image. Based on these data, surface height mapping is performed in order to obtain an image gradient, which is used to fit an ellipse on the particle edges (Fig. 3). The maximum and minimum diameters of the ellipse are further used to calculate the average aspect ratio $(37,64)$.

Size and shape descriptors such as length, width, equivalent circumference and diameter of a circle, sphericity and aspect ratio provide accurate information that can be relevant for process development and monitoring and may be used as a PAT tool.

McAuliffe et al. (37) applied particle size measurement to monitoring the roller compaction process by utilizing traditional sieve analysis, and additionally a Camsizer ${ }^{\circledR}$ and Eyecon ${ }^{\circledR}$ 3D particle characterizer (Fig. 3). Eyecon ${ }^{\circledR}$ is a flash imaging technique that uses number-based size distributions, whereas Camsizer ${ }^{\circledR}$ can use both number- and volumebased dynamic imaging techniques. The use of all three methods resulted in a broadly similar trend in particle size distributions, although the absolute values varied due to dif- 


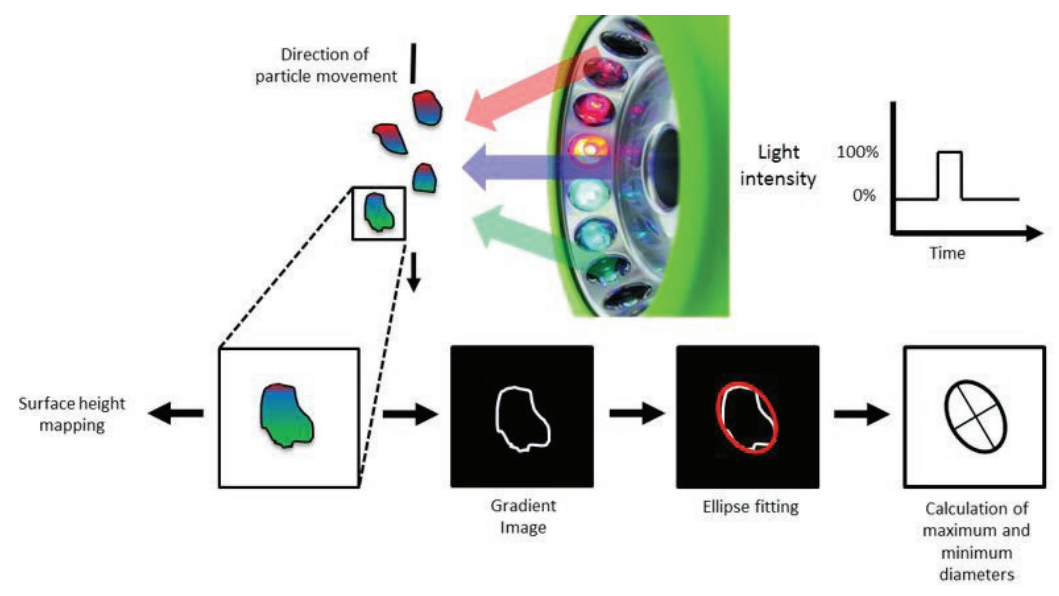

Fig. 3. Schematic presentation of the Eyecon ${ }^{\circledR}$ equipment working principle $(64)^{3}$.

ferences in measurement principles. Eyecon ${ }^{\circledR}$ gave larger values for the finer fraction (d10) and smaller values for the larger fractions (d50 and d90) compared to sieving and Camsizer ${ }^{\circledR}$ measurements. This could have occurred because Eyecon ${ }^{\circledR}$ may not have been capable of viewing individual particles if they are not fully separated, which indicates the importance of a good sampling procedure during PSD measurement.

The results show a good relationship between traditionally used in-process control method sieve analysis and novel methods for PSD measurement, and so Eyecon ${ }^{\circledR}$ and Camsizer ${ }^{\circledR}$ show significant promise as in-line PAT tools.

Further studies are needed to confirm the applicability of these tools on different formulations and to demonstrate that the methods are capable of monitoring PSD in-line during the milling process on a pilot- and industrial-scale roller compactors. Other PSD methods, such as FBRM and SFV, should also be investigated because they have been successfully utilized in monitoring PSD in other pharmaceutical operations containing milling.

\section{CONCLUSIONS}

In the author's opinion, NIR spectroscopy, NIR-CI, MRT and direct imaging techniques will be implemented as quality control in the industrial roller compaction process to monitor the manufacturing process and for identifying the root cause of manufacturing problems.

Reprinted from European Journal of Pharmaceutics and Biopharmaceutics, Vol. 85, A. F. Silva, A. Burgggraeve, Q. Denon, P. Van der Meeren, N. Sandler, T. Van Den Kerkhof, M. Hellings, C. Vervaet, J. P. Temon, J. A. Lopes and T. De Beer, Particle sizing measurements in pharmaceutical applications: comparison of in-process methods versus off-line methods, 1006-1018, 2013, with permission from Elsevier. 
However, none of the PAT methods described have been used at a commercial scale so far and, therefore, further studies with the method and process analysis optimization are necessary. Such studies should focus on the method's ability to provide fast and accurate results in order to ensure real-time monitoring and control of the process.

Overcoming the limitations of the individual technique's discussed at the end of each section of this article, the tools presented could become part of the predictive control strategy and quality control tools for continuously operating manufacturing lines involving roller compaction.

Acknowledgments. - The authors would like to thank the company Krka, d.d., Novo mesto for providing support during the writing of this article.

\section{REFERENCES}

1. R. W. Miller, Roller Compaction Technology, in Handbook of Pharmaceutical Granulation Technology (Ed. D. M. Parikh), $2^{\text {nd }}$ ed., Taylor \& Francis Group, LLC Boca Raton 2005, pp. 159-190.

2. P. J. Sheskey and R. W. Miller, Roller Compaction Technology for the Pharmaceutical Industry, in Encyclopedia of Pharmaceutical Technology (Ed. J. Swarbrick), $3^{\text {rd }}$ ed., Informa Healthcare USA, Inc., New York 2007, pp. 3159-3176.

3. Y. A. Yusof, A. C. Smith and B. J. Briscoe, Roll compaction of maize powder, Chem. Eng. Sci. 60 (2005) 3919-3931; https://doi.org/10.1016/j.ces.2005.02.025

4. Y. Teng, Z. Qui and H. Wen, Systematical approach of formulation and process development using roller compaction, Eur. J. Pharm. Biopharm. 73 (2009) 219-229; https://doi.org/10.1016/j.ejpb.2009.04.008

5. D. Z. Božič, R. Dreu and F. Vrečer, Influence of dry granulation on compactibility and capping tendency of macrolide antibiotic formulation, Int. J. Pharm. 357 (2008) 44-54; https://doi.org/10.1016/j. ijpharm.2008.01.023

6. P. D. Daugherity and J. H. Chu, Investigation of serrated roll surface differences on ribbon thickness during roller compaction, Pharm. Dev. Technol. 12 (2007) 603-608; https://doi.org/10.1080/ 10837450701563012

7. P. Kleinebudde, Roll compaction/dry granulation: pharmaceutical applications, Eur. J. Pharm. Biopharm. 58 (2004) 317-326; https://doi.org/10.1016/j.ejpb.2004.04.014

8. S. Ingelbrecht and J. P. Remon, Roller compaction and tableting of microcrystalline cellulose/drug mixtures, Int. J. Pharm. 161 (1998) 215-224; https://doi.org/10.1016/s0378-5173(97)00356-6

9. R. T. Dec, A. Zavalingos and J. C. Cunningham, Comparision of various modeling methods for analysis of powder compaction in roller press, Powder Technol. 130 (1-3) (2003) 265-271; https://doi. org/10.1016/s0032-5910(02)00203-6

10. M. Turkoglu, I. Aydin, M. Murray and A. Sakr, Modeling of a roller compaction process using neural networks and genetic algorithms, Eur. J. Pharm. Biopharm. 48 (1999) 239-245; https://doi.org/10.1016/ s0939-6411(99)00054-5

11. J. R. Johanson, A rolling theory of granular solids, J. Appl. Mech. 32 (1965) 842-848; https://doi.org/ $10.1115 / 1.3627325$

12. J. L. P. Soh, N. Boersen, M. T. Carvajal, K. R. Morris, G. E. Peck and R. Pinal, Importance of raw material attributes for modeling ribbon and granule properties in roller compaction: multivariate analysis on roll gap and NIR spectral slope as process critical control parameters, J. Pharm. Innov. 2 (2007) 106-124; https://doi.org/10.1007/s12247-007-9013-z

13. J. L. P. Soh, F. Wang, N. Boersen, R. Pinal, G. E. Peck and M. T. Carvajal, J. Cheney, H. Valthorsson and J. Pazdan, Utility of multivariate analysis in modeling the effects of raw material properties and 
A. Dular Vovko and F. Vrečer: Process analytical technology tools for process control of roller compaction in solid pharmaceuticals manufacturing, Acta Pharm. 70 (2020) 443-463.

operating parameters on granule and ribbon properties prepared in roller compaction, Drug Dev. Ind. Pharm. 34 (2008) 1022-1035; https://doi.org/10.1080/03639040801925990

14. FDA (Food Drug Administration), Guidance for Industry: PAT - A Framework for Innovative Pharmaceutical Development, Manufacture and Quality Assurance (2004).

15. T. De Beer, A. Burggraeve, M. Fonteyne, L. Saerens, J. P. Remon and C. Vervaet, Near infrared and Raman spectroscopy for the in-process monitoring of pharmaceutical production processes, Int. J. Pharm. 417 (2011) 32-47; https://doi.org/10.1016/j.ijpharm.2010.12.012

16. J. Lupayaert, D. L. Massart, Y. V. Heyden, Near-infrared spectroscopy applications in pharmaceutical analysis, Talanta 72 (2007) 865-883; https://doi.org/10.1016/j.talanta.2006.12.023

17. G. Reich, Near infrared spectroscopy and imaging: basic principles and pharmaceutical applications, Adv. Drug Deliv. Rev. 57 (2005) 1109-1143; https://doi.org/10.1016/j.addr.2005.01.020

18. K. Korasa and F. Vrečer, Overview of PAT process analysers applicable in monitoring of film coating unit operations for manufacturing of solid oral dosage forms, Eur. J. Pharm. Sci. 111 (2018) 278-292; https://doi.org/10.1016/j.ejps.2017.10.010

19. K. Korasa and F. Vrečer, A Study on the applicability of multiple process analysers in the production of coated pellets, Int. J. Pharm. (2019) (in press); https://doi.org/10.1016/j.ijpharm.2019.01.069

20. B. F. MacDonald and K. A. Prebble, Some applications of near-infrared reflectance analysis in the pharmaceutical industry, J. Pharm. Biomed. Anal. 11 (1993) 1077-1085; https://doi.org/10.1016/07317085(93)80085-f

21. M. Blanco, J. Coello, H. Iturriaga, S. Maspoch and C. de la Pezuela, Near-infrared spectroscopy in the pharmaceutical industry, The Analyst 123 (1998) 135R-150R; https://doi.org/10.1039/a802531b

22. K. Korasa, G. Hudovornik and F.Vrečer, Applicability of near-infrared spectroscopy in the monitoring of film coating and curing process of the prolonged release coated pellets, Eur. J. Pharm. Sci. 93 (2016) 484-492; https://doi.org/10.1016/j.ejps.2016.08.038

23. T. Rajalahti and O. M. Kvalheim, Multivariate data analysis in pharmaceutics: a tutorial review, Int. J. Pharm. 417 (2011) 280-290; https://doi.org/10.1016/j.ijpharm.2011.02.019

24. A. Gupta, G. E. Peck, R. W. Miller and K. R. Morris, Nondestructive measurements of the compact strength and the particle-size distribution after milling of roller compacted powders by near-infrared spectroscopy, J. Pharm. Sci. 93 (2004) 1047-1053; https://doi.org/10.1002/jps.20003

25. J. D. Kirsch and J. K. Drennen, Nondestructive tablet hardness testing by near-infrared spectroscopy: A new and robust spectral best fit algorithm, J. Pharm. Biomed. Anal. 19 (1999) 351-362; https://doi. org/10.1016/s0731-7085(98)00132-0

26. M. Donoso, D. O. Kildsig and E. S. Ghaly, Prediction of tablet hardness and porosity using near-infrared diffuse reflectance spectroscopy as a non-destructive method, Pharm. Dev. Technol. 8 (2003) 357-366; https://doi.org/10.1081/pdt-120024689

27. S. M. Short, R. P. Cogdill, P. L. D. Wildfong, J. K. Drennen and C. A. Anderson, A near-infrared spectroscopic investigation of relative density and crushing strength in four component compacts, J. Pharm. Sci. 98 (2009) 1095-1109; https://doi.org/10.1002/jps.21473

28. A. Gupta, G. E. Peck, R. W. Miller and K. R. Morris, Influence of ambient moisture on the compaction behavior of microcrystalline cellulose powder undergoing uni-axial compression and roller-compaction: A comparative study using near-infrared spectroscopy, J. Pharm. Sci. 94 (2005) 2301-2313; https://doi.org/10.1002/jps.20430

29. G. Hudovornik, K. Korasa and F. Vrečer, A study on the applicability of in-line measurements in the monitoring of the pellet coating process, Eur. J. Pharm. Sci. 75 (2015) 160-168; https://doi.org/10.1016/j. ejps.2015.04.007

30. A. J. O'Neil, R. D. Jee and A. C. Moffat, Measurement of the cumulative particle size distribution of microcrystalline cellulose using near-infrared reflectance spectroscopy, The Analyst 124 (1999) 33-36; https://doi.org/10.1039/a807134i 
A. Dular Vovko and F. Vrečer: Process analytical technology tools for process control of roller compaction in solid pharmaceuticals manufacturing, Acta Pharm. 70 (2020) 443-463.

31. M. C. Pasikatan, J. L. Steele, C. K. Spillman and E. Haque, Near infrared reflectance spectroscopy for online particle size analysis of powder and ground materials, J. Near Infrared Spectrosc. 9 (2001) 153164; https://doi.org/10.1255/jnirs.303

32. E. N. Lewis, J. W. Schopplrei, E. Lee and L. H. Kidder, NIR chemical imaging as a process analytical tool, Innovations Pharm. Technol. (2006) 107-111.

33. S. Kukec, G. Hudovornik, R. Dreu and F. Vrečer, Study of granule growth kinetics during in situ fluid bed melt granulation using in-line FBRM and SFT probes, Drug Dev. Ind. Pharm. 40 (2013) 952959; https://doi.org/10.3109/03639045.2013.791832

34. M. Khorasani, J. M. Amigo, P. Bertelsen, C. C. Sun and J. Rantanen, Process optimization of dry granulation based tableting line: extracting physical material characteristics from granules, ribbons and tablets using near-IR (NIR) spectroscopic measurement, Powder Technol. 300 (2016) 120-125; https://doi.org/10.1016/j.powtec.2016.03.004

35. D. Acevedo, A. Muliadi, A. Giridhar, J. D. Litster and R. J. Romañach, Evaluation of three approaches for real-time monitoring of roller compaction with near-infrared spectroscopy, AAPS PharmSciTech 13 (2012) 1005-1012; https://doi.org/10.1208/s12249-012-9825-0

36. M. E. Crowley, A. Hegarty, M. A. P. McAuliffe, G. E. O’Mahony, L. Kiernan, K. Hayes and A. M. Crean, Near-infrared monitoring of roller compacted ribbon density: Investigating sources of variation contributing to noisy spectral data, Eur. J. Pharm. Sci. 102 (2017) 103-114; https://doi.org/10.1016/j. ejps.2017.02.024

37. M. A. P. McAuliffe, G. E. O’Mahony, C. A. Blackshields, J. A. Collins, D. P. Egan, J. Kiernan, E. O’Neill, S. Lenihan, G. M. Walker and A. M. Crean, The use of PAT and off-line methods for monitoring of roller compacted ribbon and granule properties with a view to continuous processing, Org. Process Res. Dev. 19 (2015) 158-166; https://doi.org/10.1021/op5000013

38. A. Gupta, G. E. Peck, R. W. Millerand K. R. Morris, Real-time near-infrared monitoring of content uniformity, moisture content, compact density, tensile strength, and Young's modulus of roller compacted powder blends, J. Pharm. Sci. 94 (2005) 1589-1597; https://doi.org/10.1002/jps.20375

39. A. K. Samanta, A. D. Karande, K. Y. Ng and P. W. S. Heng, Application of near-infrared spectroscopy in real-time monitoring of product attributes of ribbed roller compacted flakes, AAPS PharmSciTech 14 (2013) 83-100; https://doi.org/10.1208/s12249-012-9890-4

40. P. V. Quyet, A. K. Samanta, C. V. Liew, L. W. Chan and P. W. S. Heng, A prediction model for monitoring ribbed roller compacted ribbons, J. Pharm. Sci. 102 (2013) 2667-2678; https://doi.org/10.1002/ jps.23635

41. R. Kona, R. M. Fahmy, G. Claycamp, J. E. Polli, M. Martinez and S. W. Hoag, Quality-by-Design III: Application of near-infrared spectroscopy to monitor roller compaction in-process and product quality attributes of immediate release tablets, AAPS PharmSciTech 16 (2015) 202-216; https://doi. org/10.1208/s12249-014-0180-1

42. T. Feng, F. Wang, R. Pinal, C. Wassgren and M. T. Carvajal, Investigation of the variability of NIR in-line monitoring of roller compaction process by using fast Fourier transform (FFT) analysis, AAPS PharmSciTech 9 (2008) 419-424; https://doi.org/10.1208/s12249-008-9054-8

43. H. Lim, V. S. Dave, L. Kidder, E. N. Lewis, R. Fahmy and S. W. Hoag, Assessment of the critical factors affecting the porosity of roller compacted ribbons and the feasibility of using NIR chemical imaging to evaluate the porosity distribution, Int. J. Pharm. 410 (2011) 1-8; https://doi.org/10.1016/j. ijpharm.2011.02.028

44. J. D. Kirsch and J. K. Drennen, Near-infrared spectroscopy: Applications in the analysis of tablets and solid pharmaceutical dosage, Appl. Spectrosc. Rev. 30 (1995) 139-174; https://doi.org/10.1080/ 05704929508000906

45. J. M. Amigo, Practical issues of hyperspectral imaging analysis of solid dosage forms, Anal. Bioanal. Chem. 398 (2010) 93-109; https://doi.org/10.1007/s00216-010-3828-z 
A. Dular Vovko and F. Vrečer: Process analytical technology tools for process control of roller compaction in solid pharmaceuticals manufacturing, Acta Pharm. 70 (2020) 443-463.

46. F. C. Clarke, S. V. Hammond, R. D. Jee and A. C. Moffat, Determination of the information depth and sample size for the analysis of pharmaceutical materials using reflectance near-infrared microscopy, Appl. Spectrosc. 56 (2002) 1475-1483; https://doi.org/10.1366/00037020260377797

47. P. Prajapati, R. Solanki, V. Modi and T. Basuri, A brief review on NIR spectroscopy and its pharmaceutical applications, Int. J. Pharm. Chem. Anal. 3 (2016) 117-123; https://doi.org/10.5958/23942797.2016.00018.6

48. H. Dalvi, C. Fauteux-Lefebvre, J. M. Guay, N. Abatzoglou and R. Gosselin, Concentration monitoring with near infrared chemical imaging in a tableting press, J. Spectral Imaging 7 (2018) 1-18; https://doi. org/10.1255/jsi.2018.a5

49. N. Souihi, D. Nilsson, M. Josefson and J. Trygg, Near-infrared chemical imaging (NIR-CI) on roll compacted ribbons and tablets - multivariate mapping of physical and chemical properties, Int. J. Pharm. 483 (2015) 200-211; https://doi.org/10.1016/j.ijpharm.2015.02.006

50. P. Guigon and O. Simon, Roll press design - influence of force feed system on compaction, Powder Technol. 130 (2003) 41-48; https://doi.org/10.1016/s0032-5910(02)00223-1

51. M. Khorasani, J. M. Amigo, J. Sonnergaard, P. Olsen, P. Bertelsen and J. Rantanen, Visualization and prediction of porosity in roller compacted ribbons with near-infrared chemical imaging (NIR-CI), J. Pharm. Biomed. Anal. 109 (2015) 11-17; https://doi.org/10.1016/j.jpba.2015.02.008

52. M. Khorasani, J. M. Amigo, C. C. Sun, P. Bertelsen and J. Rantanen, Near-infrared chemical imaging (NIR-CI) as a process monitoring solution for a production-line of roll compaction and tableting, Eur. J. Pharm. Biopharm. 93 (2015) 293-302; https://doi.org/10.1016/j.ejpb.2015.04.008

53. N. Souihi, M. Josefson, P. Tajarobi, B. Gururajan and Johan Trygg, Design space estimation of the roller compaction process, Ind. Eng. Chem. Res. 52 (2013) 12408-12419; https://doi.org/10.1021/ie303580y

54. A. Gupta, J. Austin, S. Davis, M. Harris and G. Reklaitis, A novel microwave sensor for real-time online monitoring of roll compacts of pharmaceutical powders online - a comparative case study with NIR, J. Pharm. Sci. 104 (2015) 1787-1794; https://doi.org/10.1002/jps.24409

55. R. Knöchel, W. Taute and C. Döscher, Stray field ring resonators and a novel trough guide resonator for precise microwave moisture and density measurements, Meas. Sci. Technol. 18 (2007) 1061-1068; https://doi.org/10.1088/0957-0233/18/4/014

56. S. Trabelsi, A. W. Kraszewski and S. O. Nelson, A microwave method for on-line determination of bulk density and moisture content of particulate materials, IEEE Trans. Instrum. Meas. 47 (1998) 127132; https://doi.org/10.1109/19.728804

57. C. C. Corredor, D. Bu and D. Both, Comparison of near infrared and microwave resonance sensors for at-line moisture determination in powders and tablets, Anal. Chim. Acta 696 (2011) 84-93; https:// doi.org/10.1016/j.aca.2011.03.048

58. C. Buschmüller, W. Wiedey, C. Döscher, J. Dressler and J. Breitkreutz, In-line monitoring of granule moisture in fluidized-bed dryers using microwave resonance technology, Eur. J. Pharm. Biopharm. 69 (2008) 380-387; https://doi.org/10.1016/j.ejpb.2007.09.014

59. V. Lourenço, T. Herdling, G. Reich, J. C. Menezes and D. Lochmann, Combining microwave resonance technology to multivariate data analysis as a novel PAT tool to improve process understanding in fluid bed granulation, Eur. J. Pharm. Biopharm. 78 (2011) 513-521; https://doi.org/10.1016/j.ejpb.2011.02.008

60. J. Austin, A. Gupta, R. McDonnell, G. V. Reklaitis and M. T. Harris, The use of near-infrared and microwave resonance sensing to monitor a continuous roller compaction process, J. Pharm. Sci. 102 (2013) 1895-1904; https://doi.org/10.1002/jps.23536

61. R. Keintz, G. Fariss and P. Okoye, Thermal effusivity and power consumption as PAT tools for monitoring granulation end point, Pharm. Technol. 30 (2006) 60-72.

62. Y. Roy, N. Mathis, S. Closs, J. Boodram, M. Hervas, Sundarajan, T. Marason and W. Meyer, Online thermal effusivity monitoring: a promising technique for determining when to conclude blending of magnesium stearate, Tablets $\mathcal{E}$ Capsules 3 (2005) 38-47. 
63. M. K. Ghorab, R. Chatlapalli, S. Hasan and A. Nagi, Application of thermal effusivity as a process analytical technology tool for monitoring and control of the roller compaction process, AAPS PharmSciTech 8 (2007) E155-E161; https://doi.org/10.1208/pt0801023

64. A. F. Silva, A. Burgggraeve, Q. Denon, P. Van der Meeren, N. Sandler, T. Van Den Kerkhof, M. Hellings, C. Vervaet, J. P. Temon, J. A. Lopes and T. De Beer, Particle sizing measurements in pharmaceutical applications: comparison of in-process methods versus off-line methods, Eur. J. Pharm. Biopharm. 85 (2013) 1006-1018; https://doi.org/10.1016/j.ejpb.2013.03.032

65. W. Yu and B. C. Hancock, Evaluation of dynamic image analysis for characterizing pharmaceutical excipient particles, Int. J. Pharm. 361 (2008) 150-157; https://doi.org/10.1016/j.ijpharm.2008.05.025

66. V. R. Nalluri, P. Schirg, X. Gao, A. Virdis, G. Imanidis and M. Kuentz, Different modes of dynamic image analysis in monitoring of pharmaceutical dry milling process, Int. J. Pharm. 391 (2010) 107-114; https://doi.org/10.1016/j.ijpharm.2010.02.027

67. R. Xu and O. A. Di Guida, Comparison of sizing small particles using different technologies, Powder Technol. 132 (2003) 145-153; https://doi.org/10.1016/s0032-5910(03)00048-2 